\title{
Bright light therapy for symptoms of anxiety and depression in focal epilepsy: randomised controlled trial
}

\author{
Sallie Baxendale, John O'Sullivan and Dominic Heaney
}

\section{Background}

Bright light therapy is an effective treatment for seasonal affective disorder and non-seasonal depression. Depression and anxiety are common psychiatric comorbidities in epilepsy.

\section{Aims}

To examine the efficacy of bright light therapy for symptoms of anxiety and depression in adults with focal epilepsy (trial registration at ClinicalTrials.gov: NCT01028456).

\section{Method}

We recruited 101 adults with medically intractable focal epilepsy. Participants completed the Hospital Anxiety and Depression scale (HADS) at the beginning $\left(T_{1}\right)$ and end of a 12-week baseline period $\left(T_{2}\right)$ and again after 12 weeks of daily light therapy $\left(T_{3}\right)$, with 51 participants using a highintensity light box and 50 using a low-intensity one. Seizure diaries were kept throughout the baseline and trial period.

\section{Results}

A total of 58 patients completed the trial. Anxiety and depression scores were significantly reduced following the light therapy at $T_{3}$ in both the high- and low-intensity groups.

\section{Conclusions}

Light therapy resulted in a significant reduction in symptoms of anxiety and depression but we did not find any differences between high- $v$. low-intensity treatment. This may, therefore, be an effective treatment for symptoms of low mood in epilepsy at lower intensities than those typically used to treat seasonal affective disorder. Further work is needed to investigate this possibility with an adequate placebo condition.

\section{Declaration of interest}

None.
Depression is the most common comorbidity of epilepsy and there is a close bidirectional relationship between the two conditions. People with epilepsy have increased incidence rates of depression and anxiety in the 3 years before the onset of their first seizure and subsequent epilepsy diagnosis. These findings suggest common underlying pathophysiological mechanisms that both lower seizure threshold and increase risk for mood disturbance. ${ }^{1,2}$ Treatments for the two conditions also overlap. Some anti-epileptic medications are also used to treat mood disorders and vagal nerve stimulation, originally developed as a treatment for medically intractable seizures, is currently being investigated as a new treatment for depression. ${ }^{3,4}$ Many people with epilepsy are reluctant to add to their burden of medication to treat mood disorders, particularly those with medically intractable epilepsy who are often already taking three or more anti-epileptic drugs. There is therefore a pressing clinical need for effective non-pharmacological treatments for mood disorders in this group. Bright light therapy suppresses melatonin secretion in humans ${ }^{5}$ and is an effective treatment for seasonal affective disorder. ${ }^{6,7}$ It is also proving to be a promising treatment for non-seasonal depression ${ }^{8,9}$ and is increasingly being tested and applied in a variety of other conditions with some efficacy reported in the treatment of sleep disorders, ${ }^{10}$ dementia $^{11}$ and insulin regulation in diabetes mellitus. ${ }^{12}$ We conducted a randomised controlled trial (RCT) of bright light therapy as a therapy for the symptoms of anxiety and depression in adults with medically intractable focal epilepsy. We hypothesised that it would have a beneficial effect on symptoms of anxiety and depression in patients with medically intractable epilepsy.

\section{Method}

The primary outcome measure of the trial was seizure control (trial registration at ClinicalTrials.gov: NCT01028456). The efficacy of bright light therapy for seizure control in this patient sample have been reported separately. ${ }^{13}$ This paper describes the efficacy of bright light therapy on the secondary outcome measures of anxiety and depression in the sample. The trial methods and design have been described previously in accordance with the CONSORT statement ${ }^{14}$ guidelines for reporting the results of RCTs. ${ }^{13}$ Modifications and deviations from the registered protocol are presented in the online supplement.

\section{Trial design}

This was a single-centre, balanced (1:1) double-blind, controlled, parallel group study conducted in the UK (Fig. 1). Following a 12-week baseline monitoring period (14 September 2010 to 6 December 2010), participants received either therapeutic highor low-intensity doses of light therapy from a cosmetically identical device during the 12 -week treatment phase of the study (4 January 2011 to 28 March 2011).

The participants were randomised to the high-intensity/lowintensity treatment arm on receipt of the completed consent form using an automated permuted block randomisation with a block size of 10 and an allocation ratio of 1:1. ${ }^{15}$ Both senior investigators who conducted the statistical analyses and wrote up the results were masked to the code throughout the study and at the time of data analyses. The participants were masked as to whether they had received a high- or low-intensity light box. The boxes are identical externally and the difference in luminance is only clear if they are turned on and examined side by side. Since each participant only saw their own light box they would not know whether it was set at a high- or low-intensity output.

The seasonal hours of daylight in the baseline and treatment trial conditions were matched, with the 12 -week baseline and treatment phases equally balanced 2 weeks either side of the winter 


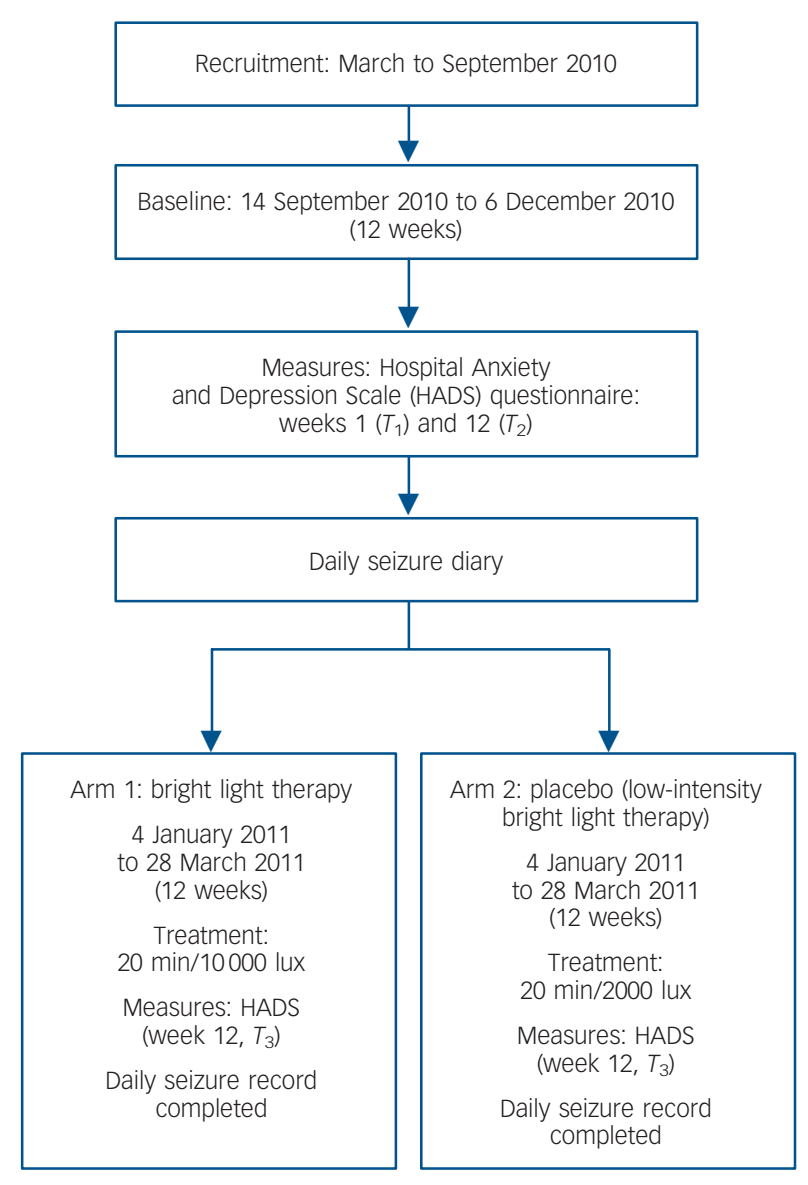

Fig. 1 Study design.

equinox on the 21 December. Participants completed a daily seizure diary during the baseline and treatment phases of the trial. Participants completed the Hospital Anxiety and Depression Scale $(\mathrm{HADS})^{16}$ at the beginning and end of the baseline period of monitoring (September 2010 and December 2010). They completed the questionnaire again at the end of the treatment phase (March 2011, Fig. 1).

The majority of the questionnaires were completed by the participants in their homes. Some were completed by the participants at the hospital during clinic visits. At the design stage of the trial we assumed an average score of 9 (s.d. =4) on the HADS scales completed at baseline. A total of 60 participants (30 in each condition) is sufficient to provide $90 \%$ statistical power to detect a reduction in the HADS score of three points or more. Ethical approval for the study was granted by The National Hospital for Neurology \& Neurosurgery and Institute of Neurology Joint Research Ethics Committee in December 2009 (ref 09/H0716/68).

\section{Participants}

Participants were recruited directly from the epilepsy clinics at the National Hospital for Neurology and Neurosurgery in London, UK. Adult patients (over the age of 18) with medically intractable, focal epilepsy, who reported an average of four seizures or more a month in the clinic were eligible to participate in the trial. Patients who had undergone previous epilepsy surgery and who continued to experience seizures were not excluded. Patients with an underlying progressive neurological disorder and those who were not able to give informed consent to participate in the study were excluded. In total 101 participants were recruited to the trial and 58 returned full questionnaire data for analyses $(n=27$ in the lowintensity group; $n=31$ high-intensity group). In the high-intensity group, $15.6 \%(n=8)$ of participants failed to return their HADS questionnaires. In the low-intensity group, 22\% $(n=11)$ failed to return their completed questionnaires (Fisher's exact test, not significant, $P>0.05$ ). Patients who failed to return the full set of questionnaires did not differ from those who returned completed questionnaires in terms of their age, or initial levels of anxiety or depression reported at the beginning of the study $\left(T_{1}\right)$. There was no gender bias in the patients who failed to return their questionnaires (Fisher's exact test $P>0.05$ ). Figure 2 shows patient losses through the trial and Table 1 details the baseline demographic and clinical characteristics of the group.

\section{Interventions: bright light therapy}

The Diamond 5 Light Box, supplied by Full Spectrum Lighting UK Ltd, was used to provide the therapy. In the treatment condition these lamps emitted 10000 lux at $61 \mathrm{~cm}$ (approximately arms length), with a treatment time of $20 \mathrm{~min}$. In the lowintensity arm of the trial the lamps were modified to emit 2000 lux at $61 \mathrm{~cm}$. The lamp still appeared very bright (in the absence of the 10000 lux comparison) but the light emitted should have been at subtherapeutic levels according to the seasonal affective disorder literature. Participants in both the high-intensity and low-intensity arms of the trial were instructed to use their light box every morning for 20-30 min, within an hour of waking up, during the treatment phase of the trial. Although all patients were instructed to use the light box every day, practical constraints (travel, changes in routine, etc.) meant that this was not always possible. Therefore during the treatment phase, the participants were asked to record how many days they actually used the light box.

\section{Statistical analyses}

Factorial repeated measures ANOVA were used to examine the effects of bright light therapy in the high- and low-intensity groups on the self-reported measures of anxiety and depression collected at the beginning of the baseline period in September $2010\left(T_{1}\right)$, at the end of the baseline period, in December 2010 $\left(T_{2}\right)$ and at the end of the treatment period, March/April $2011\left(T_{3}\right)$.

\section{Results}

\section{Bright light therapy tolerability}

The high-intensity group used their light boxes for an average of 79.5 days (s.d. $=6.5$, maximum 84 , minimum 54) over the 12 -week period of the trial and the low-intensity group used them for an average of 77.9 days (s.d. $=9.2$, maximum 84 , minimum 40). There were no significant differences between the high- and low-intensity groups in the number of hours that they used their light boxes over the trial period $(t=0.77$, d.f. $=56, P>0.05)$. The high- and low-intensity groups did not differ in the number of seizures they reported during the 12-week trial $(t=-1.8$, d.f. $=56, P>0.05)$ or the extent of seizure reduction they reported compared with baseline measures $(t=-0.56, P>0.05)$. Full details of the efficacy of bright light therapy for seizure control in this group have been previously published. ${ }^{13}$

\section{Anxiety}

For the anxiety scores, Mauchly's test indicated that the assumption of sphericity had been violated for the main effect of time of assessment $\left(\chi^{2}=9.2\right.$, d.f. $\left.=2, P=0.01\right)$ therefore degrees of freedom were corrected using Greenhouse-Geisser estimates of sphericity. There was a significant effect of the time of assessment at 


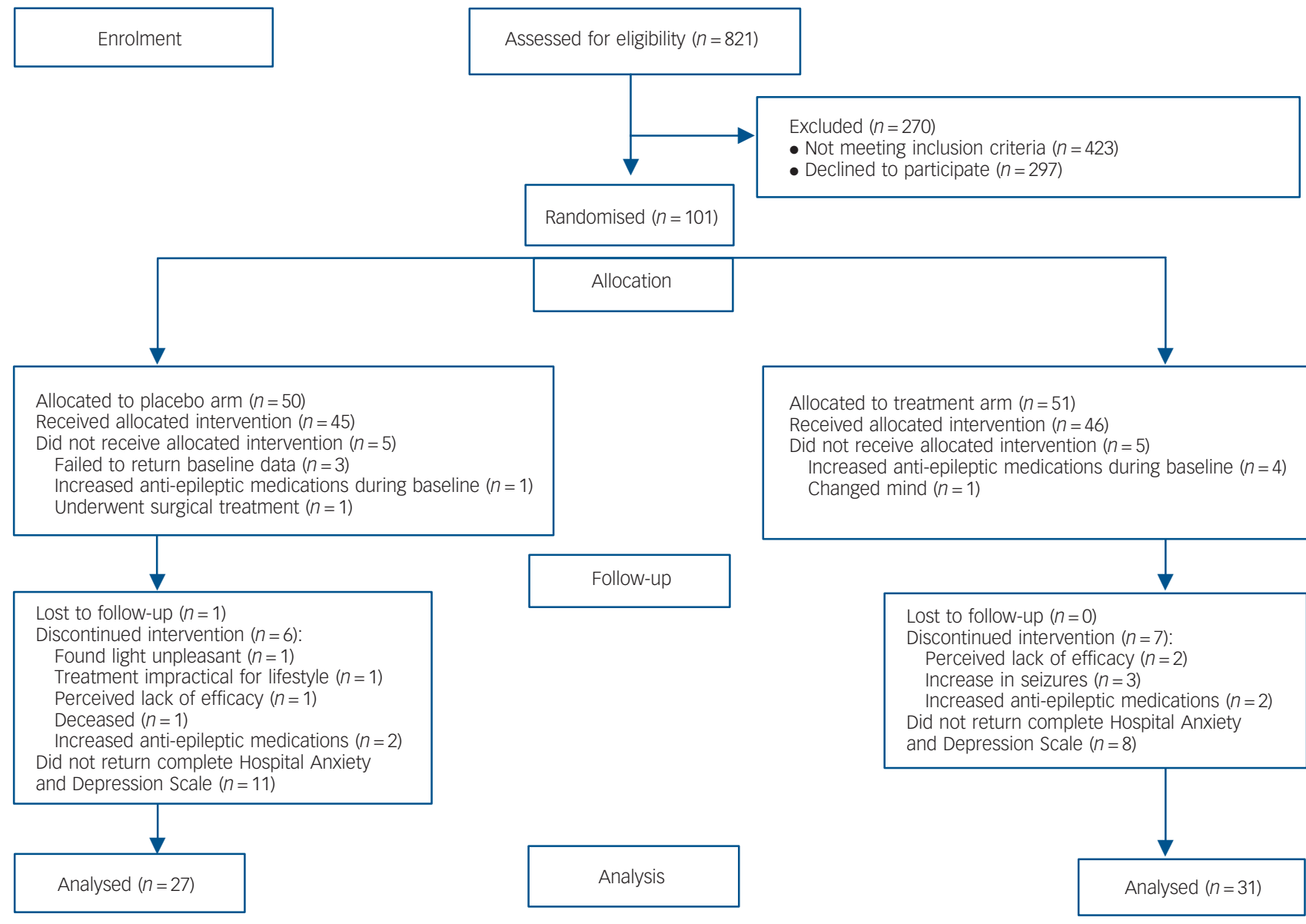

Fig. 2 Participant flow diagram.

Table 1 Baseline demographic and clinical characteristics of the group

Low-intensity group $(n=27)$

High-intensity group $(n=31)$

\begin{tabular}{|c|c|c|}
\hline Age, years: mean (s.d.) & 42.9 (11.9) & $46.6(12.8)$ \\
\hline \multicolumn{3}{|l|}{ Gender, $n$} \\
\hline Male & 11 & 13 \\
\hline Female & 16 & 18 \\
\hline \multicolumn{3}{|l|}{ Epilepsy, $n$} \\
\hline Temporal lobe focus & 18 & 24 \\
\hline Frontal lobe focus & 4 & 3 \\
\hline Other $^{a}$ & 5 & 4 \\
\hline
\end{tabular}

$P<0.01$ on the self-reported levels of anxiety on the HADS $(F=5.9$, d.f. $=1.7, P<0.01)$. Post hoc contrasts revealed that the anxiety scores were significantly lower at the end of the treatment phase $\left(T_{3}\right)$ than the two earlier assessments $\left(T_{1}\right.$ and $\left.T_{2}\right)$ in both the high- and low-intensity groups. There was no significant difference between the measures of anxiety at $T_{1}$ and $T_{2}$. There was no significant interaction between group and time of assessment on the HADS anxiety score $(F=08$., d.f. $=1.7, P>0.05)$ (Fig. 3$)$.

\section{Depression}

Mauchly's test indicated that the assumption of sphericity was not violated for the main effect of time of assessment on the depression scores $\left(\chi^{2}=4.6\right.$, d.f. $\left.=2, P>0.05\right)$. There was a significant effect of the time of assessment at $P<0.001$ on the self-reported levels of depression on the HADS $(F=8.9$, d.f. $=2)$. Contrasts revealed that the depression scores were significantly lower for both the high- and low-intensity groups at the end of the baseline phase $\left(T_{2}\right)$ compared with the levels reported at the beginning of the study $\left(T_{1}\right)(t=2.1$, d.f. $=57, P=0.04)$. A further significant decrease in depression scores was reported at the end of the treatment phase $\left(T_{3}\right)$ in both groups $(t=3.0$, d.f. $=57, P<0.01)$. There was no significant interaction between group and time of assessment on the HADS depression score $(F=1.3$, d.f. $=2$, $P>0.05$ ) (Fig. 4).

\section{Changes in clinically significant levels of anxiety and depression}

At the beginning of the trial 16 patients in the high-intensity group and 10 in the low-intensity group scored eight or more on the anxiety scale of the HADS, indicating clinically significant 


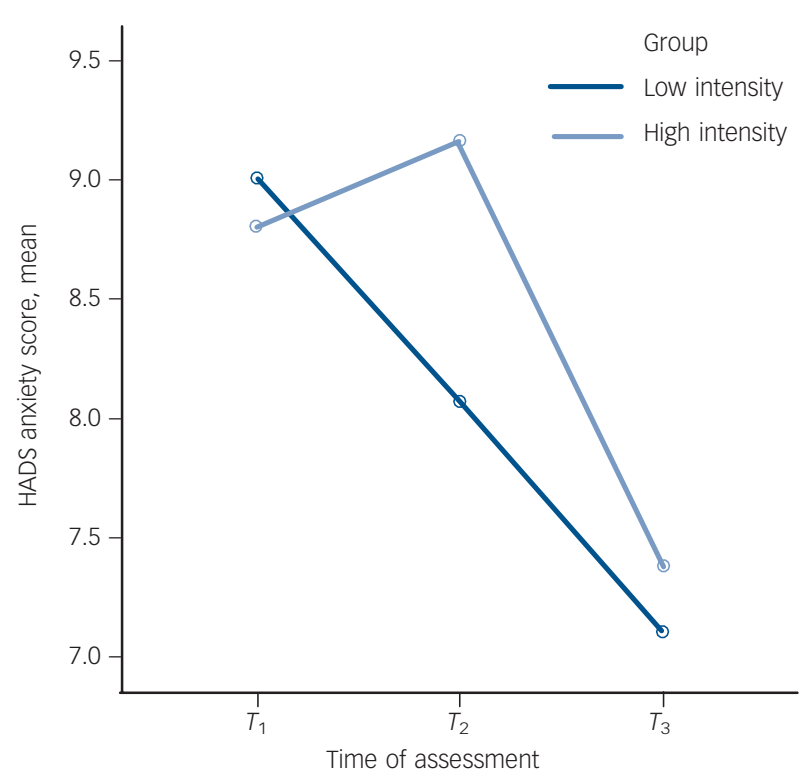

Fig. 3 Mean Hospital Anxiety and Depression Scale (HADS) anxiety scores at $T_{1}, T_{2}$ and $T_{3}$ for the high- and low-intensity light groups.

$T_{1}$, beginning of baseline (September 2010); $T_{2}$, end of baseline (December 2010); $T_{3}$, end of treatment (April 2011).

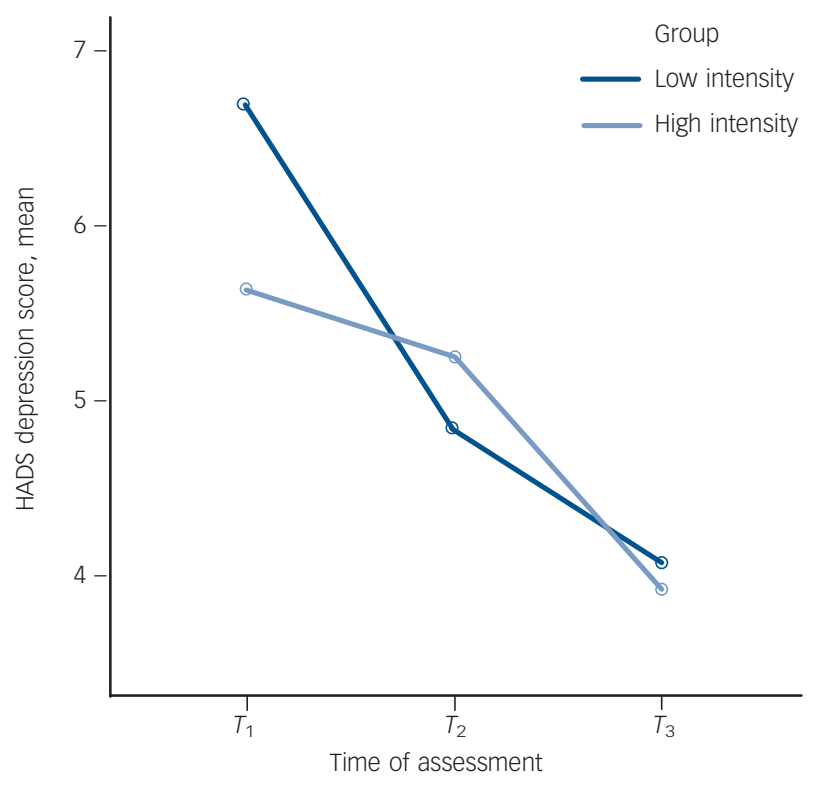

Fig. 4 Mean Hospital Anxiety and Depression Scale (HADS) depression scores at $T_{1}, T_{2}$ and $T_{3}$ for the high- and low-intensity light groups.

$T_{1}$, beginning of baseline (September 2010); $T_{2}$, end of baseline (December 2010); $T_{3}$, end of treatment (April 2011). levels of anxiety. At the end of the trial, 9 of the 16 patients (56\%) in the high-intensity group had reduced their scores to eight or below on the HADS compared with 2 of the $10(20 \%)$ in the low-intensity group $\left(\chi^{2}=3.31\right.$, d.f. $\left.=1, P<0.05\right)$.

At the beginning of the trial six patients in the high-intensity group and seven in the low-intensity group scored eight or more on the depression scale of the HADS, indicating clinically significant symptoms of depression. At the end of the trial, four of the six patients $(66 \%)$ in the high-intensity group had reduced their scores to eight or below compared with three of the seven $(43 \%)$ in the low-intensity group $\left(\chi^{2}=0.12\right.$, d.f. $\left.=1, P>0.05\right)$.

\section{Mood and seizure control}

There were no significant correlations between the number of seizures (partial, generalised and total) recorded over the baseline period and measures of anxiety at $T_{1}$ and $T_{2}$. However, depression scores at $T_{1}$ were significantly correlated with the number of drop attacks recorded over the baseline period (Pearson correlation $0.31, P=0.01)$. In the treatment phase of the study, measures of anxiety and depression at $T_{2}$ and $T_{3}$ were not significantly correlated with the number of seizures recorded (partial, generalised or total) at $T_{2}$ or $T_{3}$. In addition there were no significant correlations between the change in seizure frequency from $T_{2}$ to $T_{3}$ (seizure reduction) and changes in self-reported measures of anxiety and depression over the same period.

\section{Discussion}

Although there was a significant reduction in the severity of selfreported symptoms of both anxiety and depression at the end of the trial, these reductions were observed in both the high- and low-intensity patient groups. There were no significant differences in the HADS scores of patients in the low- $v$. high-intensity arms of the trial. Reductions in anxiety and depression were not related to changes in seizure frequency over the course of the trial. This is not surprising since it is often the diagnosis of epilepsy itself and the concomitant unpredictability of seizure occurrence that are associated with elevated levels of anxiety and depression in this population, not the number of seizures experienced.

At face value, these results suggest that bright light therapy is no more effective than a placebo in reducing symptoms of anxiety and depression in people with epilepsy. However, a number of features in the data suggest that there may be some value in pursuing this line of enquiry further. Although levels of depression significantly reduced over the baseline period $(P<0.04)$, the reduction was significantly more marked over the subsequent trial period $(P<0.01)$. Given the decline in depression scores over the baseline period probably derived from the placebo effects associated with being enrolled on a trial with weekly contact from the researchers, one would not expect a further, steeper decline in the latter half of the trial when the contact routines were well established. It is unlikely that relaxation during the light therapy sessions had a significant effect on the patients as they had to keep their eyes open during the session and were encouraged to use the light while carrying out routine tasks in the morning (including eating breakfast and checking emails). However, exposure to bright light in the morning can help to regulate the sleep-wake cycle, as can a fixed morning routine, and these factors may have had a significant clinical effect on measures of mood in both the high- and low-intensity groups, independent of the intensity of the light they were exposed to.

These results prompt an examination of the placebo condition employed. We employed a 2000 lux light box as the placebo condition in this trial on the basis of the dose-response relationship in bright light therapy established in the treatment of seasonal affective disorder. Exposure to 2000-2500 lux typically requires treatment times of at least $2 \mathrm{~h}$ to provide a therapeutic effect on low mood in seasonal affective disorder populations. The dose-response relationship in seasonal affective disorder has guided much of the research and clinical practice in bright light therapy, ${ }^{17,18}$ with greater intensities of light now being used for 
shorter duration in clinical populations. However, lower intensities of light have proved effective in reducing symptoms in some patients with less severe symptoms in seasonal affective disorder, ${ }^{19}$ albeit at longer durations than employed in this study. It is possible that lower intensities of light are required in different clinical populations, such as people with epilepsy, who may present with less severe symptoms of mood disorder than those typically seen in seasonal affective disorder samples. If this is the case, our placebo condition, the low-intensity bright light therapy, may have been more powerful than we intended.

The difficulties of creating an effective placebo that keeps the participants masked to the treatment arm to which they have been allocated in a study of bright light therapy have been previously discussed. ${ }^{13}$ One solution may be to utilise light from different parts of the spectrum (for example red light $v$. blue light) following the findings from studies that have suggested it may be exposure to light within a relatively narrow spectrum, rather than overall luminance, that may be the trigger for the therapeutic effects seen in conditions that respond to the regulation of melatonin. ${ }^{20-22}$

The numbers of patients in our sample who presented with clinically significant levels of anxiety and depression were small. The finding that high-intensity light therapy may be beneficial in reducing the symptoms of anxiety in this group is encouraging, but further work will be needed to confirm these preliminary results, given the small sample size in this study. All of the patients who participated in this study had medically intractable epilepsy and experienced frequent seizures. The psychosocial and psychiatric characteristics of this population will be different from the majority of people with epilepsy whose seizures are fully controlled by medication. Caution should therefore be employed when generalising the results from this study to the wider population of people with epilepsy.

\section{Future directions}

We acknowledge that these findings could be interpreted in a number of ways. Although it is possible that our placebo light was actually a clinically effective intensity for bright light therapy in this patient population, it is also possible that the effects we observed were as a result of factors unrelated to the intensity of the light emitted by the light boxes, including chance, seasonal variation and improved regulation of the sleep-wake cycle. Further work with an adequate placebo condition and a control group given other morning tasks might help to elucidate the mechanisms that may underlie any clinical response. Although the numbers of patients with clinically significant levels of anxiety and depression in this study were relatively small, the initial findings are encouraging. Again, further trials would be needed to establish a therapeutic effect in this population.

Sallie Baxendale, BSC (Hons), MSC, PhD, CPsychol, Department of Clinical and Experimental Epilepsy, UCL Institute of Neurology, Department of Neuropsychology, National Hospital for Neurology \& Neurosurgery, London and Epilepsy Society, Chalfont St Peter; John O'Sullivan, BSC, UCLH NHS Hospitals NHS Trust, London; Dominic Heaney, MB BCh, MRCP, PhD, National Hospital for Neurology and Neurosurgery and UCLH NHS Hospitals NHS Trust and the UCL Institute of Neurology, London, UK

Correspondence: Sallie Baxendale, Department of Neuropsychology (Box 37), National Hospital for Neurology \& Neurosurgery, Queen Square, London WC1N 3BG, UK. Email: s.baxendale@ucl.ac.uk

First received 7 Oct 2012, final revision 2 Jan 2013, accepted 5 Feb 2013

\section{Funding}

This study was funded by a research grant number AP1202 from Action Medical Research,

\section{References}

1 Hesdorffer DC, Ishihara L, Mynepalli L, Webb DJ, Weil J, Hauser WA. Epilepsy, suicidality, and psychiatric disorders: a bidirectional association. Ann Neurol 2012; 72: 184-91.

2 Hesdorffer DC, Hauser WA, Olafsson E, Ludvigsson P, Kjartansson O. Depression and suicide attempt as risk factors for incident unprovoked seizures. Ann Neurol 2006; 59: 35-41.

3 Cristancho P, Cristancho MA, Baltuch GH, Thase ME, O'Reardon JP. Effectiveness and safety of vagus nerve stimulation for severe treatmentresistant major depression in clinical practice after FDA approval: outcomes at 1 year. J Clin Psychiatry 2011; 72: 1376-82.

4 Kosel M, Brockmann H, Frick C, Zobel A, Schlaepfer TE. Chronic vagus nerve stimulation for treatment-resistant depression increases regional cerebral blood flow in the dorsolateral prefrontal cortex. Psychiatry Res 2011; 191: 153-9.

5 Lewy AJ, Wehr TA, Goodwin FK, Newsome DA, Markey SP. Light suppresses melatonin secretion in humans. Science 1980; 210: 1267-9.

6 Lam RW, Levitt AJ, Levitan RD, Enns MW, Morehouse R, Michalak EE, et al. The Can-SAD study: a randomized controlled trial of the effectiveness of light therapy and fluoxetine in patients with winter seasonal affective disorder. Am J Psychiatry 2006; 163: 805-12.

7 Westrin A, Lam RW. Seasonal affective disorder: a clinical update. Ann Clin Psychiatry 2007; 19: 239-46.

8 Golden RN, Gaynes BN, Ekstrom RD, Hamer RM, Jacobsen FM, Suppes T, et al. The efficacy of light therapy in the treatment of mood disorders: a review and meta-analysis of the evidence. Am J Psychiatry 2005; 162: 656-62.

9 Tuunainen A, Kripke DF, Endo T. Light treatment for non-seasonal depression. Cochrane Database Syst Rev 2004; 2: CD004050.

10 Shirani A, St Louis EK. Illuminating rationale and uses for light therapy J Clin Sleep Med 2009; 5: 155-63.

11 Burns A, Allen H, Tomenson B, Duignan D, Byrne J. Bright light therapy for agitation in dementia: a randomized controlled trial. Int Psychogeriatr 2009; 21: 711-21.

12 Allen NH, Kerr D, Smythe PJ, Martin N, Osola K, Thompson C. Insulin sensitivity after phototherapy for seasonal affective disorder. Lancet 1992; 339: 1065-6.

13 Baxendale S, O'Sullivan J, Heaney D. Bright light therapy as an add on treatment for medically intractable epilepsy. Epilepsy Behav 2012; 24: 359-64.

14 Schulz KF. CONSORT 2010 statement: updated guidelines for reporting parallel group randomised trials. BMJ 2010; 340: 332

15 Dallal GE. Automated permuted block randomisation: plan generator. Randomization.com, 2011 (http://www randomization com).

16 Zigmond AS, Snaith RP. The Hospital Anxiety and Depression Scale. Acta Psychiatr Scand 1983; 67: 361-70.

17 Terman M, Terman JS. Light therapy for seasonal and nonseasonal depression: efficacy, protocol, safety, and side effects. CNS Spectr 2005; 10: $647-63$.

18 Lee TM, Chan CC. Dose-response relationship of phototherapy for seasonal affective disorder: a meta-analysis. Acta Psychiatr Scand 1999; 99: 315-23.

19 Wirz-Justice A, Bucheli C, Graw P, Kielholz P, Fisch HU, Woggon B. Light treatment of seasonal affective disorder in Switzerland. Acta Psychiatr Scand 1986; 74: 193-204.

20 Berson DM, Dunn FA, Takao M. Phototransduction by retinal ganglion cells that set the circadian clock. Science 2002; 295: 1070-3.

21 Berson DM. Strange vision: ganglion cells as circadian photoreceptors. Trends Neurosci 2003; 26: 314-20.

22 Meesters $Y$, Dekker V, Schlangen $\mathrm{U}$, Bos EH, Ruiter MJ. Low-intensity blueenriched white light (750 lux) and standard bright light (10,000 lux) are equally effective in treating SAD. A randomized controlled study. BMC Psychiatry 2011; 11: 17. 